Small Methods. 2018 September 11; 2(9): . doi:10.1002/smtd.201700323.

\title{
Fluorescence Polarization Control for On-Off Switching of Single Molecules at Cryogenic Temperatures
}

\author{
Christiaan N. Hulleman, \\ Department of Imaging Physics Delft University of Technology Delft 2628, CJ, The Netherlands \\ Maximiliaan Huisman, \\ RNA Therapeutics Institute University of Massachusetts Medical School Worcester, MA 01605, \\ USA
}

Robert J. Moerland,

Department of Imaging Physics Delft University of Technology Delft 2628, CJ, The Netherlands

David Grünwald,

RNA Therapeutics Institute University of Massachusetts Medical School Worcester, MA 01605, USA

\section{Sjoerd Stallinga, and}

Department of Imaging Physics Delft University of Technology Delft 2628, CJ, The Netherlands

\section{Bernd Rieger}

Department of Imaging Physics Delft University of Technology Delft 2628, CJ, The Netherlands

\begin{abstract}
Light microscopy, allowing sub-diffraction-limited resolution, has been among the fastest developing techniques at the interface of biology, chemistry, and physics. Intriguingly no theoretical limit exists on how far the underlying measurement uncertainty can be lowered. In particular data fusion of large amounts of images can reduce the measurement error to match the resolution of structural methods like cryo-electron microscopy. Fluorescence, although reliant on a reporter molecule and therefore not the first choice to obtain ultraresolution structures, brings highly specific labeling of molecules in a large assembly to the table and inherently allows the detection of multiple colors, which enables the interrogation of multiple molecular species at the same time in the same sample. Here, the problems to be solved in the coming years, with the aim of higher resolution, are discussed, and what polarization depletion of fluorescence at cryogenic temperatures can contribute for fluorescence imaging of biological samples, like whole cells, is described.
\end{abstract}

\section{Keywords}

cryogenic; polarization; single molecules; STED; super-resolution

b.rieger@tudelft.nl.

Conflict of Interest

The authors declare no conflict of interest. 


\section{Introduction}

Over the last decade, new microscopy methods, frequently referred to as "super-resolution microscopy" or "nanoscopy," have achieved much higher resolution $(\approx 10-50 \mathrm{~nm})$ than conventional light microscopy ( $\approx 250 \mathrm{~nm}$ ). The resolution of conventional microscopy is limited by diffraction to a length scale $\lambda /(2 \mathrm{NA})$, where $\lambda$ is the emission wavelength, and $\mathrm{NA}=n \sin (a)$ is the so-called numerical aperture of the objective, where $n$ is the refractive index of the immersion medium and $a$ is the marginal ray angle of the light beam collected by the objective. With the introduction of different nanoscopy techniques ${ }^{[1-4]}$ the diffraction limit has been circumvented, giving a resolution that starts to close the resolution gap between light and electron microscopy. Where electron microscopy can reveal details below the order of a nanometer, it does not offer efficient specific labeling or multicolor imaging the way light microscopy does.

A group of techniques termed single-molecule localization microscopy (SMLM), e.g., photoactivated localization microscopy (PALM), (direct) stochastic optical reconstruction microscopy ((d)STORM), and many other flavors, ${ }^{[2-5]}$ has found the most widespread use, as it is relatively undemanding on the experimental setup and the subsequent image analysis. The key idea is to image and localize isolated single fluorescent molecules over many acquisitions. The ability of switching the fluorescent labels between a bright emitting "on" state and a dark nonemitting "off" state, resulting in blinking, is essential. This leads to a sparse subset of all labels in the sample being in their "on" state at any time point, which can be localized with a much higher precision than the diffraction limit. ${ }^{[6]}$ This localization uncertainty is on the order of $\lambda /\left(\mathrm{NA} \sqrt{\mathrm{N}_{\mathrm{ph}}}\right)$, implying that already a moderate photon count of $100-1000$ results in a ten times smaller uncertainty compared to the diffraction limit. The required ratio of on/off times to image only single emitters in a region of size $\lambda / \mathrm{NA}$ is typically smaller than $1 / 100$ to $1 / 1000$ depending on the labeling density, exposure time, and additional parameters. ${ }^{[7]}$

The image resolution, defined as the size of the smallest detail that can be reliably discerned in an image, is determined by the localization precision and the density of fluorescent labels. Previously we have introduced the concept of Fourier ring correlation (FRC) into superresolution microscopy for taking all resolution factors into account. ${ }^{[8]}$ The FRC quantifies the available image information as a function of spatial frequency, i.e., across all length scales, and points to a resolution limit via a threshold criterion. The FRC resolution in SMLM can be a value of $2 \pi$ times the localization uncertainty, depending on an adequate labeling density, ${ }^{[8]}$ providing a resolution of down to $\approx 30 \mathrm{~nm}$ for high-photon-yield fluorophores like Alexa647 (several thousand photons, localization precision of $\approx 5 \mathrm{~nm}$ ).

There are a number of emerging developments that address the limitations of labeling technologies. Advances in bio-photochemistry have resulted in the development of labels that make a direct covalent bond to the target molecule, such as dick-chemistry, ${ }^{[9,10]}$ effectively giving a small $(<1 \mathrm{~nm})$ label size. Improvements of known dyes like Rhodamines have led to much brighter, more stable, and cell-permeable labels with tunable emission properties. ${ }^{[11,12]}$ Also, techniques for high labeling density ${ }^{[13]}$ and the use of data-fusion techniques ${ }^{[14,15]}$ ameliorate the labeling limitations. Based upon these developments, it may 
be anticipated that the localization imprecision will soon become the limiting factor for resolution.

Very recently, a new breakthrough technique to further push localization imprecision was proposed, called MINFLUX. ${ }^{[16]}$ In this technique a single fluorophore is illuminated by a doughnut beam a number of times (typically four times), where the position of the doughnut beam is changed from illumination to illumination across a region of size $L$. The number of photons per illumination is collected at a photodiode and the set of photon counts is used in a triangulation procedure to estimate the position of the molecule. Surprisingly, the localization precision scales as $L / \sqrt{N}_{\mathrm{ph}}$, independent of the diffraction length $\lambda / \mathrm{NA}$. By choosing $L$ on the order of $50 \mathrm{~nm}$, an order of magnitude in precision can be gained compared to standard SMLM. The big drawback of this technique, however, is that the position of the molecule must be established to be within the tiny region of interest of size $L$ $\approx 50 \mathrm{~nm}$ by a prior experiment. A development road to application of MINFLUX across the full field of view of the objective does not appear to be in sight, and seems less than straightforward to devise.

The localization precision can be reduced to well below $1 \mathrm{~nm}$ if more than $10^{6}$ photons are recorded. Collection of these amounts of photons is possible for uncaging dyes, ${ }^{[17]}$ which today have been used infrequently, in DNA PAINT approaches where an effectively endless reservoir of dyes is imaged, ${ }^{[18]}$ or through imaging at cryogenic temperatures. ${ }^{[19-21]} \mathrm{At}$ cryogenic temperatures, dyes have a very low rate of photobleaching and localization precisions below $1 \mathrm{~nm}$ are typical. For fluorescent proteins, however, freezing only offers a moderate increase in total photon count before bleaching. ${ }^{[22]}$ The increased localization precision is especially beneficial for data-fusion techniques that rely on localizations to register or align different identical particles to obtain a super-super-resolved particle. ${ }^{[15]}$

A challenge for data-fusion methods lies in the need for the sample to be immobilized during acquisition of individual images, but working with cells at cryogenic temperatures has the inherent advantage of fixation, while the method of vitrification by plunge freezing is considered a very fast and mild fixation technique, compared to chemical fixation at room temperature. ${ }^{[23]}$ With vitrification being used for cryo-electron-microscopy (cryo-EM) sample preparation it also allows easier integration into correlative studies. ${ }^{\text {[23-25] }}$

On the downside, the traditional switching mechanism for photon emission of dyes does not work for frozen samples. Photoswitching either requires a conformational change of the fluorophore itself, such as in PALM, ${ }^{[2]}$ or a liquid buffer solution for chemical interaction with the fluorophores, such as in dSTORM. ${ }^{[4]}$ Very inefficient photoconversion at cryogenic temperatures has been reported and used for correlative studies in transmission electron microscopy, ${ }^{[22]}$ but imaging of a densely labeled sample would not have been possible at such photo-switching rates. For a very small number of dyes, spontaneous blinking has successfully been used to reconstruct a $3 \mathrm{D}$ structure from $2 \mathrm{D}$ projections. ${ }^{[20]}$ 


\section{Fluorescence Polarization Control}

Here, we propose the use of polarization control to establish on/off switching of fluorophores at cryogenic temperatures. At cryogenic temperatures, already in the range of liquid nitrogen, the absorption dipole axis of an individual fluorophore has a fixed orientation making it possible to tune the excitation of the fluorophores by rotating the state of polarization of the excitation beam (see Figure 1). The excitation efficiency scales as $\cos ^{2} \theta$ with $\theta$ being the angle between the (linear) excitation polarization and the dipole axis. [26] This effect alone gives rise to an on/off ratio of one to one, which, however, is far from what is needed for producing the sparsity that is typically used in SMLM. The level of sparsity can be improved by making use of fluorescence depletion by stimulated emission, similar to stimulated emission depletion (STED). ${ }^{[1]}$ A second beam, redshifted compared to the excitation beam, and with a polarization orthogonal to the excitation beam, is used to illuminate the sample (see Figure 1). As a result most excited molecules are driven back to the ground state with the exception of molecules with an absorption dipole that is close to the orientation perpendicular to the polarization of the depletion beam. As a consequence, the fluorescence emission will no longer follow the original $\cos ^{2} \theta$ distribution, but a much more sharply peaked distribution instead.

We can make an estimate of the improvement in sparsity by employing the analogy of the proposed method with STED. Hell[27] stated that the resolution of STED scales as

$$
\frac{\lambda}{2 \mathrm{NA} \sqrt{1+I_{\mathrm{dep}} / I_{0}}}
$$

where $I_{\mathrm{dep}}$ is the intensity of the depletion beam and $I_{0} \approx \hbar \omega / \sigma \tau$ (with $\hbar \omega$ being the photon energy, $\sigma$ the cross-section, and $\tau$ the lifetime) at constant intensity. Similarly, we conjecture that the angular emission profile after depletion has a full width at half maximum (FWHM) that may be described by

$$
\mathrm{FWHM} \approx \frac{\pi}{2 \sqrt{1+I_{\mathrm{dep}} / I_{0}}}
$$

resulting in a sparsity factor $\pi / \mathrm{FWHM}$ that can be much larger than 1 , in principle achieving the same type of FWHM narrowing as in standard STED.

So far, we have only considered excitation and depletion polarizations and dipole orientations in the plane of the sample. This matches with the relatively low NA of objective lenses that can be used in cryogenic setups (typically up to NA $=0.7$ ). ${ }^{[19,21]}$ Under these conditions the polarization of the excitation and depletion beams is necessarily close to being in-plane polarized, and fluorophores with dipole orientations that are substantially tilted with respect to the sample plane are not excited and remain invisible. Possible extensions to high-NA excitation, depletion, and imaging using immersion technology, in 
particular a solid immersion lens, would open up possibilities for polarization control over the full $4 \pi$ solid angle of polarization and dipole orientations.

Hafi et al. already put forward the idea to use polarization as an effective way to introduce sparsity in combination with STED, similar to our setup in Figure 1. ${ }^{[28]}$ Later Frahm and Keller showed however that their results were, in fact, due to post-processing of the data by a sparsity-enhancing deconvolution algorithm and not based on polarization control. ${ }^{[29]}$ Closer inspection of the data of Hafi et al. reveals that they reported very poor modulation depths when changing the polarization (their Figure 2). ${ }^{[28]}$ Two experimental factors might have caused these poor modulation depths, thereby impairing their results, while the conceptual idea is working, as we demonstrate below. To do so we first improved the effective polarization in the sample plane (the first experimental factor), by carefully calibrating the different optical elements in the microscope such that full modulation over the background can be seen. Second, we used fluorescent molecules fixed by spincoating in a polymer solution to have stable molecular transition dipoles (the second experimental factor).

\subsection{Optimizing Light Polarization at the Sample}

We developed a method to control the polarization of excitation and STED beam at the sample plane. To this end, we calibrate the optical system to know how polarization is changed by the different components in the setup as depicted in Figure 1, and, in particular, we measured the effect of the retarders (half wave plate (HWP) and quarter wave plate (QWP)) instead of assuming their nominal action. In the following, we briefly introduce the concept of the Stokes vector and the Mueller matrix, which allows the description (and manipulation) of the polarization state of light. We describe the electric field of a monochromatic plane wave, traveling along the $z$-axis, by

$$
\begin{aligned}
& E_{x}(t)=E_{0 x} \cos \left(\omega t+\delta_{x}\right) \\
& E_{y}(t)=E_{0 y} \cos \left(\omega t+\delta_{y}\right)
\end{aligned}
$$

Here, we have taken $z=0$ for convenience, and $E_{0 x}$ and $E_{0 y}$ represent the amplitude of the electric field in the $x$ - and $y$-directions, respectively. The angular frequency is given by $\omega$, and $\delta_{X}$ and $\delta_{y}$ are the phase factors for the respective electric field components. In general, the tip of the electric field vector traces an ellipse in space for arbitrary amplitudes and phases, the polarization ellipse. It can be shown that the Stokes 4-vector $\boldsymbol{S}$ and the electric fields are related as ${ }^{[31]}$ 


$$
\left(\begin{array}{c}
S_{0} \\
S_{1} \\
S_{2} \\
S_{3}
\end{array}\right)=\left(\begin{array}{c}
E_{0 x}^{2}+E_{0 y}^{2} \\
E_{0 x}^{2}-E_{0 y}^{2} \\
2 E_{0 x} E_{0 y} \cos \delta \\
2 E_{0 x} E_{0 y} \sin \delta
\end{array}\right)
$$

with $\delta=\delta_{y}-\delta_{x}$. The first stokes parameter $S_{0}$ describes the total intensity of the light. Of the remaining parameters, $S_{1}$ describes the amount of horizontally or vertically polarized light, $S_{2}$ the amount of diagonally polarized light $\left( \pm 45^{\circ}\right)$, and $S_{3}$ the amount of left/righthanded circularly polarized light. The $4 \times 4$ Mueller matrix $\mathbf{M}$ transforms two Stokes vectors $\boldsymbol{S}_{\text {out }}=\mathbf{M} \boldsymbol{S}_{\text {in }}$ and describes how the polarization state is changed by an optical system. Once the Mueller matrix of a system has been determined, the required input polarization state in order to get a desired output polarization state can be retrieved via $\mathbf{M}^{-1}$. Here, we have used a dual rotating retarder polarimeter, ${ }^{[30]}$ which consists of a polarizer, a QWP, the system under test, a second QWP, and an analyzer. The two QWPs are rotated simultaneously but with a 5:1 ratio, which gives the 16 elements of $\mathbf{M}$ after Fourier analysis of the resulting intensity signal for different angles. With the free-space requirement that $\mathbf{M}$ is the identity matrix we can calibrate the polarimeter ${ }^{[32]}$ and the orientation and retardance of the retarders. In our implementation, the QWPs (AQWP05M-600, Thorlabs) are rotated with computer-controlled rotation stages (8MPR16-1, Standa). The polarizers are of the GL10 type (Thorlabs) with an anti-reflection coating suitable for the wavelength used.

In order to measure $S_{\text {in }}$ and $S_{\text {out }}$, we use a Stokes polarimeter ${ }^{[31]}$ composed of a rotating QWP (AQWP05M-600, Thorlabs) and a polarizer (GL10, Thorlabs). Here, the retarding wave plate is rotated with a computer-controlled stage (8MPR16-1, Standa) as well. The laser light running through the system is modulated with the aid of an optical chopper wheel (MC2000-EC, Thorlabs) and detected with a silicon photodiode (DET10A, Thorlabs), after focusing with an additional lens (LB1901-B, Thorlabs). A lock-in amplifier (SR830, Stanford Research Systems) is used to demodulate the detector signal. Both types of setup are computer-controlled from within MATLAB (The MathWorks). Thus, with the combination of a Stokes and a Mueller polarimeter, we can fully determine the polarization transfer function of our microscope (see Figure 1a) and the polarization state of the light itself in the sample plane.

\subsection{Results of Polarization Control}

In order to evaluate our calibration procedure over naive usage of the nominal action of the retarders, we measured Stokes vectors for both setups (see Figure 2a-c). Without DM1 and QWP2 present (see Figure 1a), we insert $638 \mathrm{~nm}$ linearly polarized laser light into the excitation path (LDH-D-C-640, PicoQuant), where the initial linear polarization is generated with a Glan-Laser calcite polarizer (GL10, Thorlabs). First, we naively try to rotate the polarization by rotating an achromatic half-wave retarder (AHWP05M-600, Thorlabs) in steps of $5^{\circ}$ and measure the polarization state at the sample plane with Stokes vector polarimetry (compare the red solid lines in Figure 2a-c). In the coordinate system of the 
microscope, a horizontal polarization, corresponding to $S=(1,1,0,0)$, is identical to $s$ polarized light hitting the dichroic mirror DM2 (FF652-Di01, Semrock). This corresponds to $a=0^{\circ}$ in Figure 2. Vertically polarized light is identical to $S=(1,-1,0,0)$ and corresponds to $a=90^{\circ}$. From Figure 2a-c we see that these two linear polarizations are well defined with normalized $S=(1,0.981,0.013,0.023) \pm(0,2,1,1) \times 10^{-3}(1 \sigma)$ at $a=0^{\circ}$ and $S=(1$, $-0.995,-0.093,-0.005) \pm(0,0.3,2,2)-10^{-3}$ at $a=90^{\circ}$. However, for the diagonal polarizations at $a=45^{\circ}$ and $a=135^{\circ}$, corresponding to $S=(1,0, \pm 1,0)$, it is obvious that $S_{2}$ is far from (minus) unity, and in fact $S_{3}$ is near (minus) unity. Therefore, in the straightforward implementation, polarizations deviating from horizontal and vertical are increasingly elliptically polarized, even approaching circularity.

Next, we calibrate our Mueller polarimeter and measure the Mueller matrix of the excitation path by the method described elsewhere. ${ }^{[32]}$ In this case, we do not insert HWP2 and QWP2 into the path. Therefore, we obtain the Mueller matrix of the combination of the dichroic mirror DM2, lens L2 (LA1433-633, Thorlabs) and the objective (Plan Apo VC 100×/1.40, Nikon), as well as a measurement of the retardances of the used QWPs. Here, we used QWP2 as part of the Mueller polarimeter, thus obtaining the actual retardance. Furthermore, we determine the retardance of HWP2 by the method described by Goldstein. ${ }^{[31]}$ The total polarization transfer function of the excitation light is then $\mathbf{M}_{\mathrm{tot}}=\mathbf{M}_{\mathrm{mic}} \mathbf{M}_{\mathrm{HWP} 2} \mathbf{M}_{\mathrm{QWP} 2}$, where $\mathbf{M}_{\mathrm{QWP} 2}$ and $\mathbf{M}_{\mathrm{HWP} 2}$ are the Mueller matrices of the wave plates, and $\mathbf{M}_{\mathrm{mic}}$ is the Mueller matrix of DM2 with the objective. By this procedure we are able to predict the Stokes vector, and hence the polarization, of the light moving through the excitation branch including HWP2 and QWP2. Moreover, we can predict the angles of the retarders that are necessary to obtain any required polarization state in the sample plane. We use this possibility to compensate the error in polarization, induced by DM2 and the objective, in order to obtain linearly polarized light in the sample plane (compare the blue solid lines in Figure 2a-c). From visual inspection, it is clear that over the whole range of linear polarizations, the ellipticity is strongly reduced (compare the blue and red lines in Figure 2c).

We focus our attention on the diagonal polarizations, which are problematic without correction. For $a=45^{\circ}, S=(1,0.015,-0.976,-0.015) \pm(0,2,2,2) \times 10^{-3}$ and for $a=$ $135^{\circ}, S=(1,0.025,0.989,-0.029) \pm(0,2,3,1) \times 10^{-3}$, after calibration. The linearity of the polarization at these angles therefore is vastly improved. We see in Figure $2 \mathrm{c}$ that there is still some residual ellipticity with the Mueller-matrix-based compensation method. We therefore add a numerical optimization algorithm, which searches for the optimal wave plate angles to minimize $S_{3}$. The cost function returns the error between the ideal and the measured Stokes vector components

$$
\text { err }=\left|S_{1}-S_{1 m}\right|+\left|S_{2}-S_{2 m}\right|+10 \times\left|S_{3}-S_{3 m}\right|
$$

where we emphasize the error in $S_{3}$, the component which leads to ellipticity. For each angle $a$, the optimization is run (compare the green solid lines in Figure 2a-c). Ideally, in all cases $S_{3}$ is identical to zero. Visibly, $S_{3}$ is closer to zero throughout the range of $a$ after optimization. The root-mean-square error for the different schemes in Figure $2 \mathrm{c}$ shows a 
substantial improvement from 0.66 (naive rotation of HWP2: red), 0.04 (Mueller matrix: blue), and 0.01 (optimized: green).

Figure 2d shows the $S_{3}$ component of a Stokes vector measurement of just an HWP (AHWP05M-980, Thorlabs, dashed red line) with the depletion laser (Mai Tai HP $750 \mathrm{~nm}$, Spectra-Physics). When compared to the case that the DM1 (FF697-SDi01, Semrock) is installed in the depletion path (red solid line), the dramatic effect of the dichroic mirror (DM) on the ellipticity of the polarization is clearly visible. We obtained an optimized DM for the depletion path from Chroma, not only for a high reflectivity of the laser, but also for a minimal phase difference between the $s$ and $p$-components, which indeed has nearly no influence on the ellipticity of the beam (cf. the measured blue curve), and merely changes the circular component, induced by the HWP, in handedness. An additional optimization step as described above essentially eliminates this part (green curve).

We now turn to single-molecule fluorescence measurements of fixed dyes (at room temperature) as a function of polarization to investigate the performance of our calibration above. We apply and compare the naive polarization rotation scheme and the calibrated, optimized scheme. For each angle $a$, we obtain a fluorescence image with the aid of an EMCCD camera (Ixon Ultra 888, Andor), with an exposure time of $1 \mathrm{~s}$, an EM gain of 25, and a sensor temperature of $-60{ }^{\circ} \mathrm{C}$. In order to prepare the samples, a stock solution of ATTO $647 \mathrm{~N}$ (ATTO-TEC) was diluted to a final concentration of $2.8 \times 10^{-10} \mathrm{M}$ in demineralized water, mixed with $0.5 \mathrm{wt} \%$ of poly(vinyl alcohol) (PVA). Spin-coating this solution at $3000 \mathrm{rpm}$ for $1 \mathrm{~min}$ onto cleaned cover slips resulted in samples of single molecules with a randomly fixed dipole transition moment. Figure $2 \mathrm{e}-\mathrm{h}$ shows the mean fluorescence intensity of a selection of four molecules for the optimized polarization rotation scheme (blue solid lines). The background (black dashed lines) has been determined as the average of five locations without any molecules. The black arrows serve as an illustration of the in-plane dipole moment of each molecule, based on the phase of the intensity modulation. Figure $2 \mathrm{i}-1$ shows the results for the naive implementation on four other molecules. Here, we clearly see the improvement of our calibration procedure. For (near) horizontal and vertical dipole orientations, the modulation depth is comparable in both cases (nearly 100\%), as for these polarizations, the DM hardly alters the polarization. However, for diagonal transition dipole moments, the calibration approach remains near a $100 \%$ modulation depth, highlighting the superiority of our scheme, in contrast to the naive implementation where the near-circular quality of the polarization at the relevant angles $a$ results in a strong electric field component along the dipole transition moment.

\section{Cryostat Design}

Among the various methods to boost the number of available photons, cooling of the sample to cryogenic temperatures, which also fixes the dipole moments of all emitters in the sample, is ideally suited for the use of polarization STED to enable high-density labeling while maintaining emitter sparsity. However, the use of an STED beam adds a heat load not typically present in imaging at cryogenic temperatures. A very powerful cryostat design, enabling fluorescence cryo-microscopy (cryoFM) on practically any microscope platform, was presented by Li et al. ${ }^{[21]}$ Adding an additional heat source to cryoFM raises the question 
as to what can be done to improve the cooling capacity of such a cryostat, both with respect to transferring heat from the sample and maintaining imaging duration upward of $5 \mathrm{~h}$.

Different methods have been suggested to increase the cooling capacity to the sample. The modularity of the Li cryostat is of high practical value as it demands a minimum of modifications to the microscope platform, arguing to focus on increasing the tank volume, raising questions as to how to best scale the size of the heat exchange interface in the cryostat (see Figure 1 in $\mathrm{Li}$ et al. ${ }^{[21]}$ ) while maintaining the structural stability of the original design. We used finite-element simulations to optimize the cooling capacity and mechanical stability. Simulations were done in Solidworks Simulation (Premium package, Dassault Systems) using a curvature-based solid mesh with four Jacobian points and element sizes between 1 and $20 \mathrm{~mm}$. For thermal simulations about 433000 nodes, forming roughly 270 000 elements, with a maximum aspect ratio of $\approx 200$ ( $>90 \%$ of elements had a ratio of $<3$ and less than $0.5 \%$ of elements had a ratio of $>10$ ) were used. For frequency simulations, finding the first five natural frequencies and their amplitude at resonance, about 170000 nodes, forming $>100000$ elements, with a maximum aspect ratio of $\approx 48$ (>89\% of elements had a ratio of $<3$ and less than $0.6 \%$ of elements had a ratio of $>10$ ) were used. The goals for a cryostat with an increased cooling capacity (see Figure 3) are to better tolerate the use of additional external heat sources, like an STED beam, possibly increase the duration of the cooling cycle to allow for longer observation times, and maintain cooling temperatures suitable for cryoFM using liquid nitrogen while maintaining maximum vibration damping.

By increasing the number of vibrationally decoupling thermal conductivity braids between the sample holder and the heat dissipater at the bottom of the nitrogen tank (Figure 3), the maximum heat flux is effectively doubled. We increased the size of the tank by a factor of two, allowing approximately double the original cold measurement time at traditional operation. The dimensions of the parts that suspend the sample holder in vacuum were optimized to increase thermal insulation and effectively dampen low-frequency oscillations, pushing the predicted resonance modes well into the kilohertz range. Specifically, we analyzed the impact of additional mounting points for the sample holder. We found that, depending on the number of spacer rings, an increase of mounting holes from 4 to 8 will result in a $2 \mathrm{~K}$ temperature increase at the sample holder, while causing $32 \mathrm{~K}$ higher temperatures at the cooling disk. As to be expected, a higher number of mounting points yield higher resonance frequencies. Differences in the first three modes (along the $x, y$, and $z$-directions) were $<30 \%$ with resonance frequencies in the range of $1900 \mathrm{~Hz}(x, y)$ and 2600 $\mathrm{Hz} \mathrm{(z)}$ if eight mounting points were used, and $1400 \mathrm{~Hz}(x, y)$ and $1900 \mathrm{~Hz}(z)$ if four mounting points were used. The fourth and fifth modes showed differences below $1 \%$ and were in the range of $2500 \mathrm{~Hz}$. Interestingly, the shape of the spacers used (strips vs rings) was shown to be in favor of rings (yielding $>20 \%$ differences in heat conductivity, roughly scaling with surface-area differences). Taken together, optimization of the cooling capacity of a closed cryostat as described by $\mathrm{Li}$ et al ${ }^{[21]}$ should use fewer mounting holes as the stability improvements lie in a frequency range well above $1 \mathrm{kHz}$, but increased numbers of mounting holes increase heat flow substantially. While the effective heat load on the cryostat introduced by an STED beam is currently unknown, our simulations indicate no downside to increasing the thermal coupling of the sample holder by increasing the number of copper braids between sample holder sample holder mount and the heat dissipater. 
While cooling performance and the resulting imaging time remain to be assessed for STED experiments, this design should be capable of dissipating a substantial part—if not all — of the heat added by a depletion beam. Sensors that accurately register the temperature cooling stages within the cryostat can be used to examine the performance during experiments, as well as to provide an estimate of the amount of energy deposited into the sample by an external source.

\section{Outlook and Conclusion}

Minimizing the localization uncertainty and reducing the overall measurement error could lead to FRC resolution values below or in the range of the size of an individual emitter. While new measurement modalities like MINFLUX show the way to improve localization uncertainty at low photon count, increasing the photon budget by means of improved labels or cooling of the sample is already possible today. The power of data fusion to reduce the measurement error has been demonstrated by resolving structural details of nuclear pore complexes in cells using SMLM style imaging. ${ }^{[14]}$ We showed that by using Stokes and Mueller polarimetry, we were able to measure and improve the effective polarization in the sample plane. We considered design limitations to increase heat flow and cooling capacity of a modular cryostat to account for the additional heat load of an STED beam in cryoFM and argue that our measurements of the effective polarization depletion in the sample plane are the missing element to achieve nanometer-ranged FRC resolution in SMLM.

Combining single-emitter-resolution localization microscopy with biological tools (i.e., CRISPR and its derivatives) to unequivocally label specific proteins inside cells would allow the visualization of structures that have never been directly seen before such as genomic substructures, DNA/RNA interactions, and protein complex conformations.

\section{Acknowledgements}

The authors thank Weixing Li and Jörg Enderlein for helpful discussion. B.R., C.N.H., and R.J.M. acknowledge the European Research Council (Grant No. 648580) and B.R., M.H., and D.G. acknowledge the National Institutes of Health (Grant No. U01EB021238). The data used in this manuscript are available online at https://doi.org/10.4121/ uuid:37139a27-181f-45a6-9aa2-eb672eed58e0.

\section{References}

[1]. Hell SW, Wichmann J, Opt. Lett. 1994, 19, 780. [PubMed: 19844443]

[2]. Betzig E, Patterson GH, Sougrat R, Lindwasser OW, Olenych S, Bonifacino JS, Davidson MW, Lippincott-Schwartz J, Hess HF, Science 2006, 313, 1642. [PubMed: 16902090]

[3]. Rust MJ, Bates M, Zhuang X, Nat. Methods 2006, 3, 793. [PubMed: 16896339]

[4]. Heilemann M, Van de Linde S, Schüttpelz M, Kasper R, Seefeldt B, Mukherjee A, Tinnefeld P, Sauer M, Angew. Chem. 2008, 47, 6172. [PubMed: 18646237]

[5]. Klein T, Proppert S, Sauer M, Histochem. Cell Biol. 2014, 141, 561. [PubMed: 24496595]

[6]. Ober RJ, Ram S, Ward SE, Biophys.J. 2004, 86, 1185. [PubMed: 14747353]

[7]. Small AR, Biophys. J. 2009, 96, L16. [PubMed: 19167282]

[8]. Nieuwenhuizen RPJ, Lidke KA, Bates M, Leyton Puig D., Grünwald D, Stallinga S, Rieger B, Nat. Methods 2013, 10, 557. [PubMed: 23624665]

[9]. Zessin PJM, Finan K, Heilemann M, J. Struct. Biol. 2012, 177, 344. [PubMed: 22226957] 
[10]. Raulf A, Spahn CK, Zessin PJM, Finan K, Bernhardt S, Heckel A, Heilemann M, RSC Adv. 2014, 4, 30462. [PubMed: 25580242]

[11]. Grimm JB, English BP, Chen J, Slaughter JP, Zhang Z, Revyakin A, Patel R, Macklin JJ, Normanno D, Singer RH, Lionnet T, Lavis LD, Nat. Methods 2015, 12, 244. [PubMed: 25599551]

[12]. Grimm JB, Brown TA, Tkachuk AN, Lavis LD, ACS Cent. Sci. 2017, 3, 975. [PubMed: 28979939]

[13]. Legant WR, Shao L, Grimm JB, Brown TA, Milkie DE, Avants BB, Lavis LD, Betzig E, Nat. Methods 2016, 13, 359. [PubMed: 26950745]

[14]. Löschberger A, van de Linde S, Dabauvalle MC, Rieger B, Heilemann M, Krohne G, Sauer M, J. Cell Sci. 2012, 125, 570. [PubMed: 22389396]

[15]. Broeken J, Johnson H, Lidke DS, Liu S, Nieuwenhuizen RPJ, Stallinga S, Lidke KA, Rieger B, Methods Appl. Fluoresc. 2015, 3, 014003.

[16]. Balzarotti F, Eilers Y, Gwosch KC, Gynnå AH, Westphal V, Stefani FD, Elf J, Hell SW, Science 2017, 355, 606. [PubMed: 28008086]

[17]. Vaughan JC, Jia S, Zhuang X, Nat. Methods 2012, 9, 1181. [PubMed: 23103881]

[18]. Jungmann R, Steinhauer C, Scheible M, Kuzyk A, Tinnefeld P, Simmel FC, Nano Lett. 2010, 10, 4756. [PubMed: 20957983]

[19]. Weisenburger S, Jing B, Hänni D, Reymond L, Schuler B, Renn A, Sandoghdar V, ChemPhysChem 2014, 15, 763. [PubMed: 24677759]

[20]. Weisenburger S, Boening D, Schomburg B, Giller K, Becker S, Griesinger C, Sandoghdar V, Nat. Methods 2017, 14, 141. [PubMed: 28068317]

[21]. Li W, Stein SC, Gregor I, Enderlein J, Opt. Express 2015, 23, 3770. [PubMed: 25836229]

[22]. Chang Y-W, Chen S, Tocheva EI, Treuner-Lange A, Löbach S, S-Andersen L, Jensen GJ, Nat. Methods 2014, 11, 737. [PubMed: 24813625]

[23]. van Driel LF, Valentijn JA, Valentijn KM, Koning RI, Koster AJ, Eur. J. Cell Biol. 2009, 88, 669. [PubMed: 19726102]

[24]. Briegel A, Chen S, Koster AJ, Plitzko JM, Schwartz CL, Jensen GJ, Methods Enzymol. 2010, 481, 317. [PubMed: 20887863]

[25]. Kopek BG, Shtengel G, Shan Xu C., Clayton DA, Hess HF, Proc. Natl. Acad. Sci. USA 2012, 109, 6136. [PubMed: 22474357]

[26]. Ha T, Enderle T, Chemla DS, Selvin PR, Weiss S, Phys. Rev. Lett. 1996, 77, 3979. [PubMed: 10062357]

[27]. Hell SW, Science 2007, 316, 1153. [PubMed: 17525330]

[28]. Hafi N, Grunwald M, Van den Heuvel LS, Aspelmeier T, Chen J-H, Zagrebelsky M, Schütte OM, Steinem C, Korte M, Munk A, Walla PJ, Nat. Methods 2014, 11, 579. [PubMed: 24705472]

[29]. Frahm L, Keller L, Nat. Methods 2016, 13, 7. [PubMed: 26716556]

[30]. Azzam RMA, Opt. Lett. 1978, 2, 148. [PubMed: 19684668]

[31]. Goldstein DH, Polarized Light, 3rd ed., CRC Press, Boca Raton, FL, USA 2010.

[32]. Hauge PS, J. Opt. Soc. Am. 1978, 68, 1519. 
a

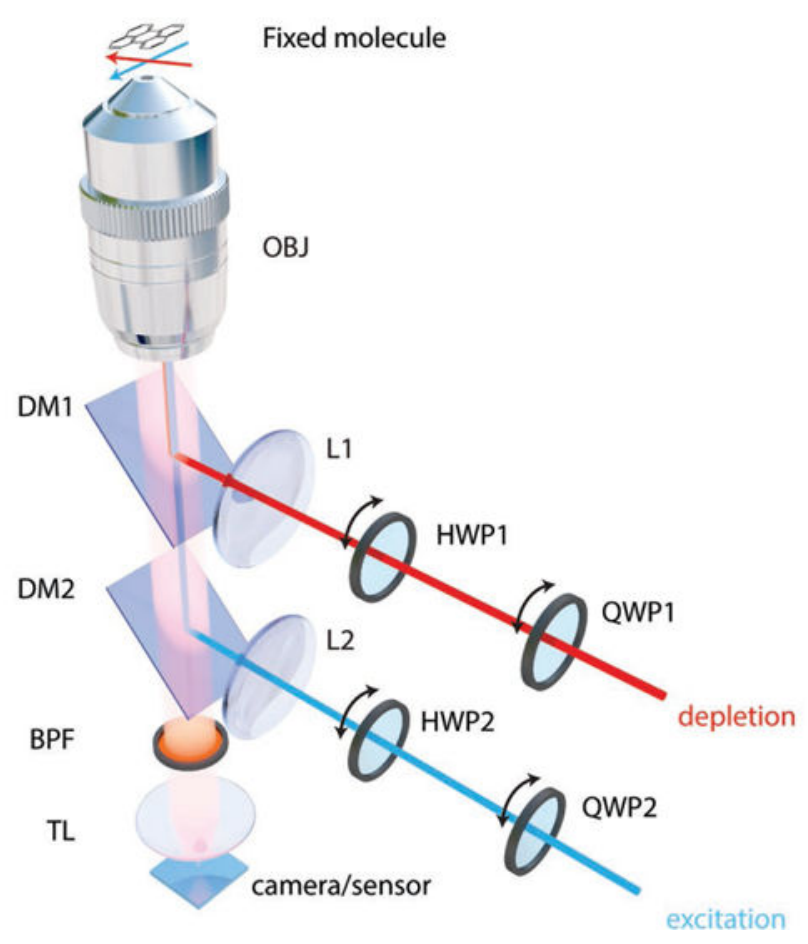

b
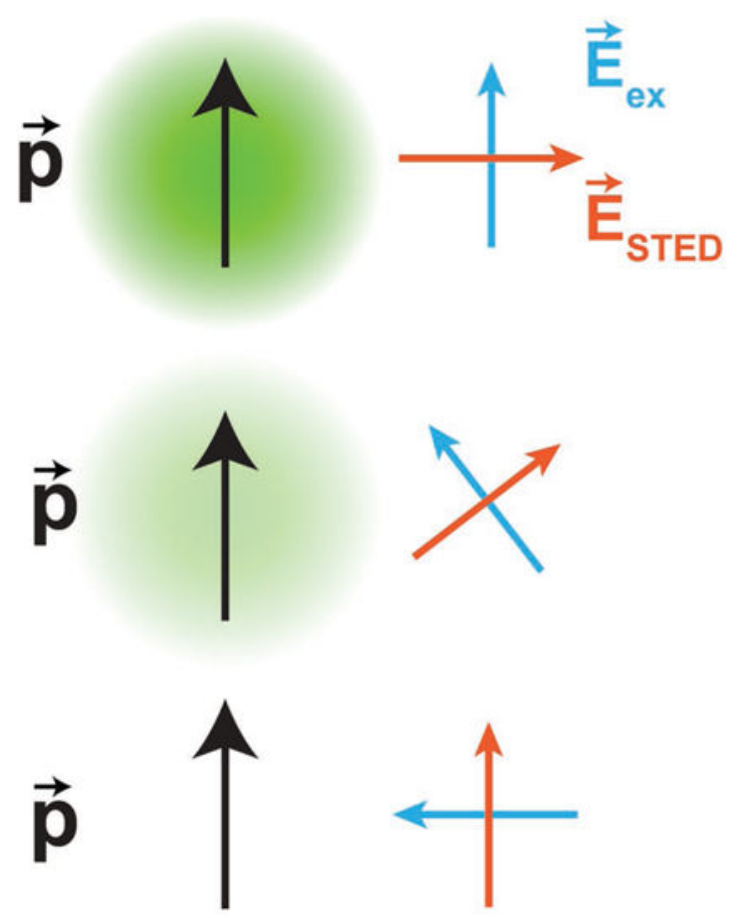

Figure 1.

a) Schematic drawing of the setup. A custom-built microscope is composed of an objective OBJ and tube lens TL. Dichroic mirrors DM1 and DM2 are used to reflect excitation and depletion laser light toward the objective, and let the fluorescence from the sample pass to the camera. A bandpass filter BPF is used to further suppress the potential leaked through laser light. Lenses L1 and L2 are used to focus the lasers on the back focal plane of the objective for epi-illumination. Full control over the polarizations of both lasers in the sample plane is obtained through the use of half-lambda (HWP1 and HWP2) and quarte r-lambda wave plates (QWP1 and QWP2) for each laser path, respectively. b) With the excitation laser $\vec{E}_{\text {ex }}$ parallel to the molecular traesition dipole $\vec{p}$ and tee depletion laser $\vec{E}_{\text {STED }}$ perpendicular, the moleeule fluoresces at maximum intensity. The excitation efficiency is reduced when $\vec{p}$ is at an angle with $\vec{E}_{\text {ex }}$. Moreover, the increased inner product with $\vec{E}_{\mathrm{STED}}$ leads to efficient depletion of the molecule and a sparsity in the sample based on angular selection of transition dipoles, compared to a case where only $\vec{E}_{\text {ex }}$ is present. 

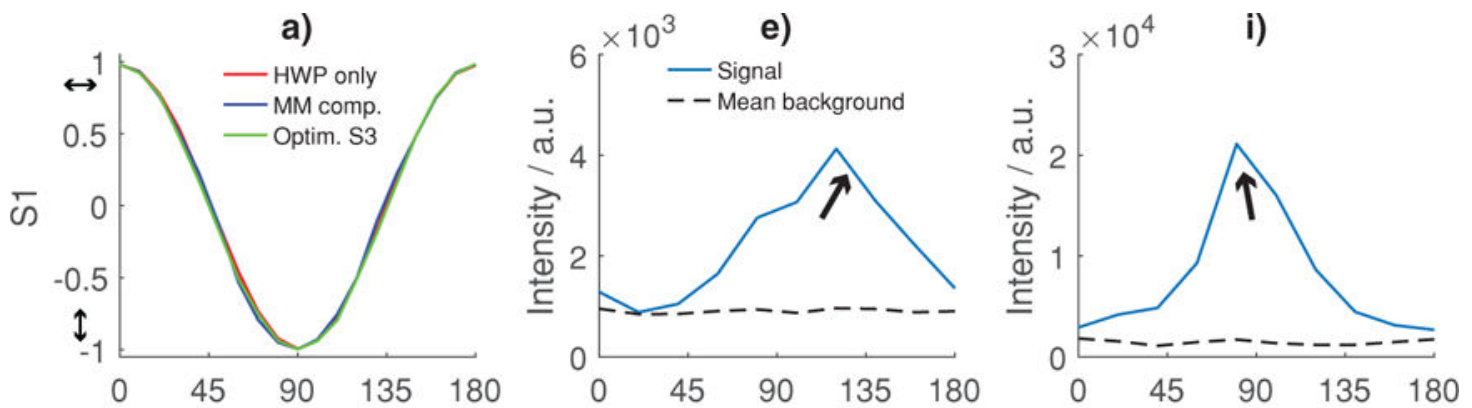

b)
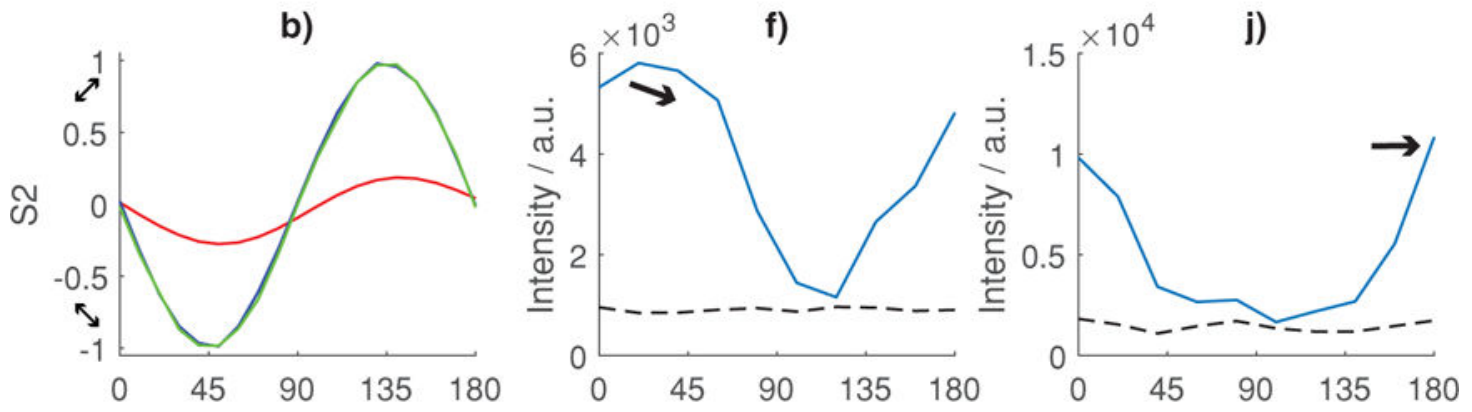

c)
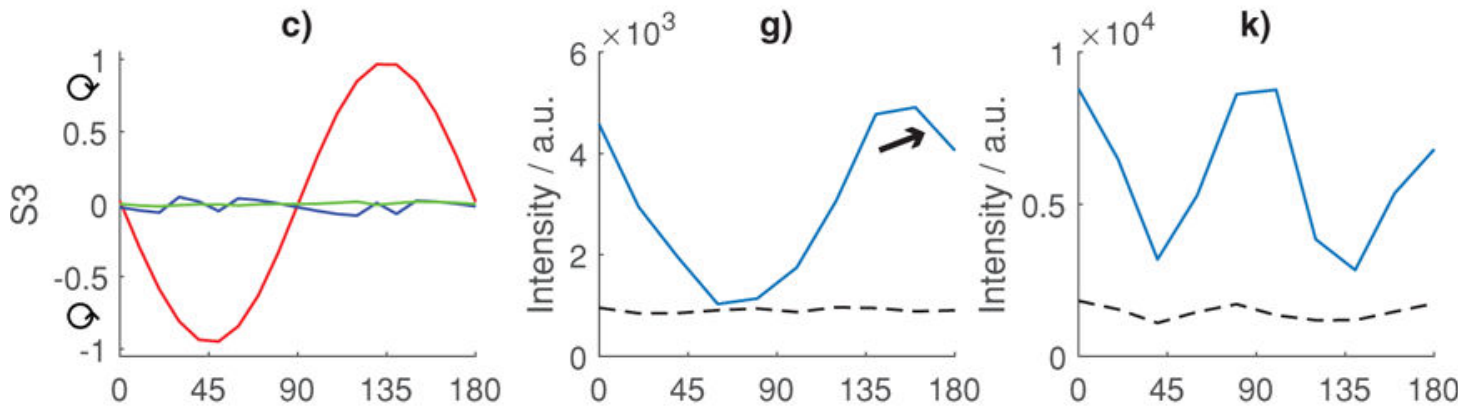

d)
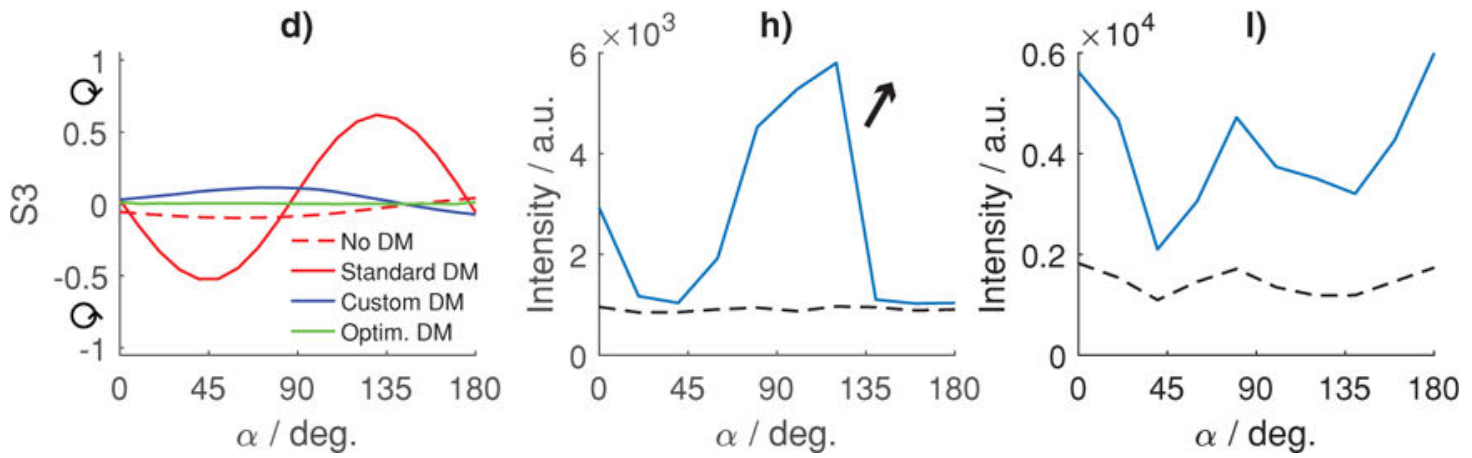

Figure 2.

a-c) Normalized Stokes vector components S1, S2, and S3 for the dichroic mirror used for excitation as a function of $a$, the desired polarization orientation angle. Rotating only an HWP yields the red curve. Using calculated orientations of the QWP and HWP based on the measured Mueller matrix yields the blue curve. Optimizing the orientation of the wave plates based on the resulting Stokes vector yields the green curve. d) Normalized S3 Stokes vector component for the standard and custom dichroic mirror for the depletion path. The dashed red curve is a direct measurement of a rotating HWP only. E-h) Mean intensity of 
single molecule emitters using the optimized QWP and HWP orientations, the black arrows depict the estimated molecule's transition orientation. I-l) Mean intensity fluctuation of single-molecule emitters during rotation of only an HWP; again the black arrows indicate estimated molecular transition dipole orientations. 


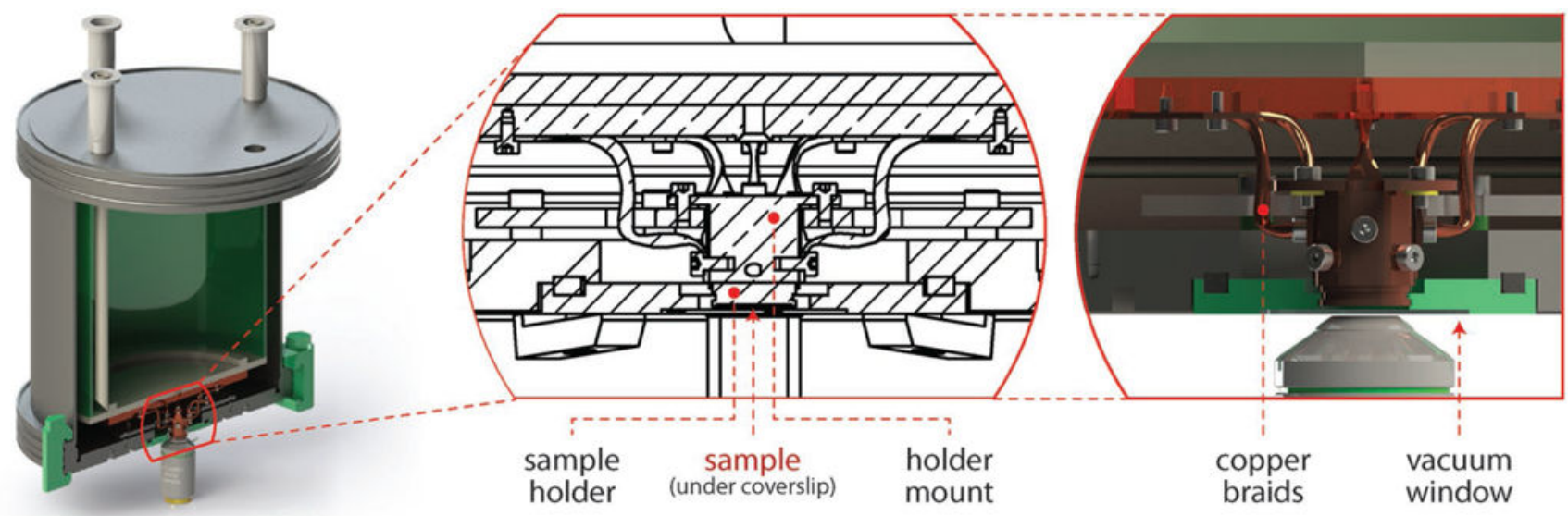

Figure 3.

Improved cryostat design for cryo-fluorescence microscopy. The model depicted here features three main improvements over the design by Li et al.: ${ }^{[21]}$ (1) a $2 \times$ larger liquid nitrogen tank for long-term super-resolution imaging, (2) four additional copper braids between the sample holder sample holder mount and the heat dissipater to allow more heat flux and increase cooling capacity, and (3) vibrational-mode optimization to maximize damping and minimize drift while maintaining thermal insulation. These improvements make the new design more suitable for cryo-polarization STED imaging. 